\title{
Blood Pressure Control in Type 2 Diabetes Mellitus
}

\author{
Tara I. Chang, MD, MS, \\ Stanford University School of Medicine, Palo Alto, California
}

Alfred K. Cheung, MD, and

Veterans Affairs Salt Lake City Healthcare System, University of Utah, Salt Lake City, Utah

Glenn M. Chertow, MD, MPH

Stanford University School of Medicine, Palo Alto, California

\begin{abstract}
Diabetes mellitus is among the developed world's most important diseases, affecting 23.6 million adults in the United States and more than 171 million adults worldwide, ${ }^{1}$ most of whom have type 2 diabetes. Cardiovascular disease is the leading cause of death in persons with diabetes mellitus. ${ }^{2}$ Because $75 \%$ of persons with diabetes mellitus have systemic hypertension, which is also a major cardiovascular risk factor, a high level of attention to blood pressure (BP) control in this population is essential. Treatment with antihypertensive agents in persons with type 2 diabetes has decreased the risk of cardiovascular events and death, ${ }^{3-5}$ and the Seventh Report of the Joint National Committee on Prevention, Detection, Evaluation, and Treatment of High Blood Pressure (JNC 7) recommends targeting BPs to $<130 / 80 \mathrm{~mm} \mathrm{Hg}$ in persons with diabetes. ${ }^{6}$ However, evidence supporting the 130/80 mm $\mathrm{Hg}$ target is scant. Of the handful of randomized trials in patients with type 2 diabetes and hypertension, ${ }^{3,4,7}$ no prior trial has achieved average BPs $<130 / 80 \mathrm{~mm} \mathrm{Hg}$, and trial conclusions have been mixed.
\end{abstract}

\section{WHAT DOES THIS IMPORTANT STUDY SHOW?}

The ACCORD (Action to Control Cardiovascular Risk in Diabetes) trial randomly assigned 10,251 individuals from 77 clinical sites in the United States and Canada with type 2 diabetes at high risk of cardiovascular events to intensive or standard glycemic therapy. ${ }^{8}$ Of these participants, 5,518 were randomly assigned to receive simvastatin plus fenofibrate or simvastatin plus placebo (the ACCORD Lipid trial), ${ }^{9}$ whereas the remaining 4,733 participants were enrolled in the ACCORD BP trial $^{10}$ and randomly assigned to a target systolic BP $<120 \mathrm{~mm} \mathrm{Hg}$ (intensive therapy) or $<140 \mathrm{~mm} \mathrm{Hg}$ (standard therapy). According to the study design, any currently available antihypertensive drug regimen could be used to achieve the target BPs. The primary outcome for all 3 studies was the composite end point of nonfatal myocardial infarction, nonfatal stroke, or cardiovascular death.

(C) 2010 by the National Kidney Foundation, Inc. Published by Elsevier Inc. All rights reserved.

Address correspondence to Glenn M. Chertow, MD, MPH, Stanford University School of Medicine, Division of Nephrology, 780 Welch Rd, Ste 106, Palo Alto, CA 94304. gchertow@ stanford.edu.

Financial Disclosure: Because Dr Cheung is a SPRINT investigator, his institution receives grants/grants pending from NHLBI. Because Dr Chertow is a SPRINT investigator, his institution receives grants/grants pending from NHLBI through a subcontract from the University of Utah. Dr Chang declares that she has no relevant financial interests. 
The ACCORD BP trial excluded participants with a serum creatinine concentration $>1.5$ $\mathrm{mg} / \mathrm{dL}$ or protein excretion $>1.0 \mathrm{~g} / 24 \mathrm{~h}$. Mean baseline serum creatinine level was 0.9 $\mathrm{mg} / \mathrm{dL}$, corresponding to a mean estimated glomerular filtration rate (eGFR) of $91.6 \pm 28.8$ $\mathrm{mL} / \mathrm{min} / 1.73 \mathrm{~m}^{2} ;<15 \%$ of participants had an eGFR $<60 \mathrm{~mL} / \mathrm{min} / 1.73 \mathrm{~m}^{2}$. Median baseline urinary albumin-creatinine ratio was $14.3 \mathrm{mg} / \mathrm{g}$ (interquartile range, 6.9-44.8). By 4 months, average systolic BP in the intensive-therapy group was $119.3 \mathrm{~mm} \mathrm{Hg}$ (95\% confidence interval [CI], 118.9-119.7) versus $133.5 \mathrm{~mm} \mathrm{Hg}(95 \% \mathrm{CI}, 133.1-133.8)$ in the standardtherapy group. An average difference in diastolic BP of $6.1 \mathrm{~mm} \mathrm{Hg}$ was observed between the 2 groups. Similar intergroup differences were maintained for the duration of the study, which had a mean follow-up of 4.7 years. The primary outcome was observed at an annual rate of $1.87 \%$ in the intensive-therapy group compared with $2.09 \%$ in the standard-therapy group, corresponding to a hazard ratio of $0.88(95 \% \mathrm{CI}, 0.73-1.06 ; P=0.20)$. The hazard ratio for total stroke (a prespecified secondary end point) favored intensive over standard therapy $(0.59 ; 95 \% \mathrm{CI}, 0.39-0.89)$, but overall rates of stroke were low in both groups $(0.32 \%$ and $0.53 \%$ per year, respectively). There were no significant differences in the other prespecified secondary outcomes, including all-cause death.

Although overall rates were low, participants in the intensive-therapy group experienced more serious adverse events (events that were life-threatening, resulted in permanent disability, or necessitated hospitalization) than participants in the standard-therapy group (3.3\% vs $1.3 \% ; P<0.001)$. In terms of adverse kidney outcomes, the intensive-therapy group had a higher proportion of participants with eGFR $<30 \mathrm{~mL} / \mathrm{min} / 1.73 \mathrm{~m}^{2}$ compared with the standard-therapy group at the end of follow-up ( $4.2 \%$ vs $2.2 \% ; P<0.001)$. However, the intensive-therapy group had fewer participants with macroalbuminuria (albumin-creatinine ratio $2300 \mathrm{mg} / \mathrm{g} ; 6.6 \%$ vs $9.7 \% ; P=0.009$ ), and there were no differences in the incidence of end-stage renal disease or need for dialysis between the 2 groups $(2.5 \%$ vs $2.4 \% ; P=0.93)$.

\section{HOW DOES THIS STUDY COMPARE WITH PRIOR STUDIES?}

$\mathrm{BP}$ control in trials of nondiabetic participants recently has been reviewed in the American Journal of Kidney Diseases. ${ }^{11}$ Directly comparing results from the ACCORD BP trial with other randomized trials of hypertension control in patients with type 2 diabetes is challenging given differences in targeted and achieved BPs, study protocols, and definitions of primary and secondary outcomes. The ACCORD BP trial is the only randomized trial to focus exclusively on systolic BP and to target (and achieve) the lowest BPs to date. The trial in individuals with diabetes mellitus with achieved BPs closest to the ACCORD BP trial is the ABCD (Appropriate Blood Pressure Control in Diabetes) trial, which randomly assigned 470 participants with type 2 diabetes and hypertension to a goal diastolic BP $<75 \mathrm{~mm} \mathrm{Hg}$ versus 80-89 mm Hg. ${ }^{12}$ In the ABCD trial, mean achieved BPs were $132 / 78 \mathrm{~mm} \mathrm{Hg}$ in the intensive-therapy group and 138/86 mm Hg in the standard-therapy groups. Similar to the results of the ACCORD BP trial, there were no statistically significant differences in risk of cardiovascular events after 5 years of follow-up.

Other previous studies of type 2 diabetes had vastly different BP targets from the ACCORD BP trial and yielded different results. The UKPDS (United Kingdom Prospective Diabetes 
Study $)^{4}$ randomly assigned 1,148 individuals with type 2 diabetes and hypertension to target BPs $<150 / 85$ or $<180 / 105 \mathrm{~mm} \mathrm{Hg}$ and achieved mean BPs of 144/82 and 154/87 mm Hg, respectively. In contrast to the ACCORD BP trial, participants in the more intensive-therapy group in the UKPDS had a $24 \%$ (95\% CI, 8-38) lower risk of any diabetes-related macrovascular or microvascular (including kidney) event compared with participants in the less intensive-therapy group. However, only participants in the more intensive-therapy group were allowed to receive angiotensin-converting enzyme inhibitors or $\beta$-blockers by study protocol, which might have conferred additional benefit to the more intensive-therapy group. In the HOT (Hypertension Optimal Treatment) study, ${ }^{3}$ subgroup analysis of 1,501 diabetic participants randomly assigned to a diastolic BP goal $<90 \mathrm{~mm} \mathrm{Hg}$ (mean achieved $\mathrm{BP}, 144 / 85 \mathrm{~mm} \mathrm{Hg}$ ) had a 2-fold higher risk of major cardiovascular events compared with the $<80-\mathrm{mm} \mathrm{Hg}$ group (mean achieved BP, 140/81 mm Hg).

\section{WHAT SHOULD CLINICIANS AND RESEARCHERS DO?}

The ACCORD BP trial showed that participants with type 2 diabetes at high risk of cardiovascular events did not have a significant decrease in the risk of composite cardiovascular events with target systolic BP $<120 \mathrm{~mm} \mathrm{Hg}$ (which is lower than the current JNC 7 guidelines) versus $140 \mathrm{~mm} \mathrm{Hg}$, but a significant decrease in risk of stroke was observed. Based on observed absolute risk differences, 476 patients per year would need to be treated to a systolic BP $<120 \mathrm{~mm} \mathrm{Hg}$ to prevent 1 stroke, but after treating only 49 patients to this target, 1 serious adverse event would be expected. Whether the benefit warrants the risk is up to the clinical judgment of the individual practitioner. The ACCORD BP trial does not support or refute current recommended systolic BP targets in patients with type 2 diabetes of $<130 \mathrm{~mm} \mathrm{Hg}$ because the study (initiated before publication of the JNC 7) was not designed to test the appropriateness of the JNC 7 BP target. However, given the low overall event rates in the ACCORD BP trial in either randomly assigned group, along with results from the HOT study, ${ }^{3}$ targeting systolic $\mathrm{BP}$ to at least $<140 \mathrm{~mm} \mathrm{Hg}$ seems justified.

Current clinical practice guidelines for persons with chronic kidney disease (CKD) also recommend target BPs $<130 / 80 \mathrm{~mm} \mathrm{Hg}$, with stronger evidence for slowing kidney disease progression than decreasing cardiovascular disease risk. ${ }^{11,13}$ The uncertainty surrounding the fundamental issue of BP control in persons with CKD emphasizes the importance of the upcoming SPRINT (Systolic Blood Pressure Intervention Trial), sponsored by the National Heart, Lung, and Blood Institute (NHLBI), the National Institute of Diabetes and Digestive and Kidney Diseases (NIDDK), the National Institute of Neurological Disorders and Stroke (NINDS), and the National Institute on Aging (NIA). ${ }^{14}$ SPRINT and SPRINT-Senior (a substudy of the elderly hypertensive population) will randomly assign 9,250 nondiabetic hypertensive adults at risk of cardiovascular disease to systolic BP targets $<120$ versus $<140$ $\mathrm{mm} \mathrm{Hg}$ and determine the effect on incident cardiovascular events and dementia.

Importantly, SPRINT plans to enroll 4,300 (45\%) individuals with moderate CKD (eGFR $<60 \mathrm{~mL} / \mathrm{min} / 1.73 \mathrm{~m}^{2}$ ), making it the largest randomized trial of BP control on cardiovascular events in patients with CKD. A main secondary outcome for the CKD subgroup in SPRINT is progression of kidney disease. Results from SPRINT, which will follow up participants for up to 6 years, will help inform our management of hypertension, 
particularly in CKD subpopulations. At present, we can only speculate about whether the results of SPRINT in nondiabetic individuals will mirror those of the ACCORD BP trial in diabetic patients or tell a completely different story. Until then, clinicians will have to carefully weigh the perceived risks and benefits of BP targets in patients with and without diabetes, CKD, or other comorbid conditions while waiting for results from future studies to guide practice.

\section{Acknowledgments}

Drs Cheung and Chertow are SPRINT investigators. The general design of SPRINT and SPRINT-Senior are subject to change.

\section{References}

1. World Health Organization. [Accessed May 20, 2010.] Diabetes Programme, Facts and Figures. http://www.who.int/diabetes/facts/world_figures/en/

2. [Accessed May 20, 2010.] National diabetes statistics. 2007. http://diabetes.niddk.nih.gov/dm/pubs/ statistics/index.htm

3. Hansson L, Zanchetti A, Carruthers SG, et al. Effects of intensive blood-pressure lowering and lowdose aspirin in patients with hypertension: principal results of the Hypertension Optimal Treatment (HOT) randomised trial. Lancet. 1998; 351(9118):1755-1762. [PubMed: 9635947]

4. UK Prospective Diabetes Study Group. Tight blood pressure control and risk of macrovascular and microvascular complications in type 2 diabetes: UKPDS 38. BMJ. 1998; 317(7160):703-713. [PubMed: 9732337]

5. Patel A. ADVANCE Collaborative Group. Effects of a fixed combination of perindopril and indapamide on macro-vascular and microvascular outcomes in patients with type 2 diabetes mellitus (the ADVANCE trial): a randomised controlled trial. Lancet. 2007; 370(9590):829-840. [PubMed: 17765963]

6. Chobanian AV, Bakris GL, Black HR, et al. National Heart, Lung, and Blood Institute Joint National Committee on Prevention, Detection, Evaluation, and Treatment of High Blood Pressure; National High Blood Pressure Education Program Coordinating Committee. The Seventh Report of the Joint National Committee on Prevention, Detection, Evaluation, and Treatment of High Blood Pressure: the JNC 7 report. JAMA. 2003; 289(19):2560-2572. [PubMed: 12748199]

7. Estacio RO, Jeffers BW, Hiatt WR, Biggerstaff SL, Gifford N, Schrier RW. The effect of nisoldipine as compared with enalapril on cardiovascular outcomes in patients with non-insulindependent diabetes and hypertension. N Engl J Med. 1998; 338(10):645-652. [PubMed: 9486993]

8. Dluhy RG, McMahon GT. Intensive glycemic control in the ACCORD and ADVANCE trials. N Engl J Med. 2008; 358(24):2630-2633. [PubMed: 18539918]

9. ACCORD Study Group. Effects of combination lipid therapy in type 2 diabetes mellitus. N Engl $\mathbf{J}$ Med. 2010; 362(17):1563-1574. [PubMed: 20228404]

10. ACCORD Study Group. Effects of intensive blood-pressure control in type 2 diabetes mellitus. $\mathrm{N}$ Engl J Med. 2010; 362(17):1575-1585. [PubMed: 20228401]

11. Shastri S, Sarnak MJ. Blood pressure target in individuals without diabetes: what is the evidence? Am J Kidney Dis. 2010; 56(3):434-438. [PubMed: 20570424]

12. Estacio RO, Jeffers BW, Gifford N, Schrier RW. Effect of blood pressure control on diabetic microvascular complications in patients with hypertension and type 2 diabetes. Diabetes Care. 2000; 23(suppl 2):B54-64. [PubMed: 10860192]

13. National Kidney Foundation. [Accessed May 25, 2010.] K/DOQI Clinical Practice Guidelines on Hypertension and Antihypertensive Agents in Chronic Kidney Disease. 2004. http:// www.kidney.org/professionals/KDOQI/guidelines_bp/index.htm

14. National Institutes of Health. [Accessed May 20, 2010.] NIH launches multi-center clinical trial to test blood pressure strategy. http://www.nih.gov/news/health/oct2009/nhlbi-29.htm 